\title{
José Bernardo Suárez: un agente de la educación popular y su visión sobre la instrucción primaria en Chile (1867)
}

\author{
Raquel Soaje de Elías ${ }^{1}$ \\ Manuel Salas Fernández ${ }^{2}$
}

Recibido: 29 de junio de 2018 - Aprobado: 31 de agosto de 2018

\begin{abstract}
Resumen:
A mediados del siglo XIX Chile avanzaba en el camino de la consolidación de la república. Los intelectuales encontraron en la educación popular uno de los medios fundamentales para el sostenimiento del orden. La fundación de la Escuela Normal en 1842 hizo realidad el proyecto de formar un estamento docente capaz de "civilizar y moralizar". En este contexto, el artículo se propone como objetivo estudiar la figura de José Bernardo Suárez, uno de los primeros preceptores que pasó por sus aulas, a través de un informe realizado en 1867 para la revista de educación Ambas Américas, editada en Nueva York. Se indaga también en ciertos aspectos de la personalidad y vida de Suárez, vinculados con su rol agente de observador y conocedor de la realidad escolar, en un momento signado por la vigencia de una ley (1860) y de un reglamento general (1863), basales del sistema educativo. Se pretende, además, analizar sus apreciaciones acerca de la formación de profesores, el perfil de educador, los métodos de enseñanza, entre otros aspectos, interpretados desde su perspectiva de visitador de escuelas, encargado de vigilar y supervisar los avances de la política educativa de la élite gobernante de la época.
\end{abstract}

Palabras clave: ideario liberal, escuelas normales, perfil de educador, métodos de enseñanza.

\section{José Bernardo Suárez: An agent in popular education and his opinion about the primary education in Chile (1867)}

\author{
Abstract \\ In the mid-nineteenth Century, Chile was moving towards the consolidation of \\ the Republic. Intellectuals found in popular education one of the fundamental \\ means for maintaining order. Founding the Normal School in 1842 made the
}

1 Argentina. Doctora en Historia, Universidad Nacional de Cuyo, Argentina. Académica del Instituto de Historia, Universidad de los Andes, Chile. E-mail: rsoaje@uandes.cl

2 Chileno. Doctor en Historia, Universidad de Texas, Austin, Estados Unidos. Académico del Instituto de Historia, Universidad de los Andes, Chile. E-mail: msalasf@utexas.edu 
project of forming a teaching body capable of "civilizing and moralizing" a reality. In this context, the article aims to study José Bernardo Suárez, one of the first preceptors who studied in those classrooms, through a report made in 1867 for the education journal Ambas Américas (Both Americas), published in New York. It also analyzes certain aspects in the personality and life of Suárez, linked to his role as an observer and expert of the school reality, at times marked by the validity of a law (1860) and a general regulation (1863), foundations of the education system. It is also intended to analyze their findings about teacher training, the teacher profile and teaching methods, among others, interpreted from the perspective of a school supervisor responsible for monitoring and supervising progresses in the education policy of the elite of that time.

Keywords: liberal ideology, Normal Schools, teacher profile, teaching methods.

\title{
José Bernardo Suárez: um agente da educação popular e sua visão sobre a instrução primária no Chile (1867)
}

\begin{abstract}
Resumo
Em meados do século XIX, o Chile avançava no caminho da consolidação da república. Os intelectuais encontraram na educação popular um dos meios fundamentais para a manutenção da ordem. Com a fundação da Escola Normal em 1842, tornou-se realidade o projeto de formar uma categoria docente capaz de "civilizar e moralizar". Neste contexto, o artigo propõe-se como objetivo estudar a figura de José Bernardo Suárez, um dos primeiros preceptores que passou por suas salas de aula, através de um relatório realizado em 1867 para a revista de educação Ambas Américas, editada em Nova York. Indaga-se, também, em certos aspectos da personalidade e vida de Suárez, vinculados com seu papel como agente observador e conhecedor da realidade escolar, em um momento marcado pela vigência de uma lei (1860) e de um regulamento geral (1863), basais do sistema educativo. Visa, além disso, analisar suas apreciações sobre a formação de professores, o perfil de educador, os métodos de ensino, entre outros aspectos, interpretados desde sua perspectiva de visitador de escolas, encarregado de vigiar e supervisionar os avanços da política educativa da elite governante da época.
\end{abstract}

Palavras-chave: Ideário liberal, Escolas Normais, Perfil do educador, Métodos de ensino.

\section{Introducción}

Hacia mediados del siglo XIX Chile avanzaba en el camino de la consolidación de la república. Los intelectuales de la época, que miraban y admiraban los modelos extranjeros, encontraron en la educación popular uno de los medios fundamentales para el sostenimiento del orden recién creado. En este proceso surgieron voces que reclamaban la creación de escuelas públicas; no obstante, 
el avance de la instrucción primaria estatal fue lento y no exento de obstáculos. Uno de estos escollos fue la escasez de profesores. Se hacía imprescindible formar a quienes debían extender la cultura, de corte liberal, y la "civilización" a través de la escuela, transmitiendo no solo conocimientos sino muy especialmente "valores y buenas costumbres", mediante los cuales el pueblo se incorporaría al nuevo orden creado por la república (Ossa, 2017: 23-52; Egaña y Monsalve, 2006). En palabras del reputado escritor y pedagogo argentino Domingo Faustino Sarmiento, llamado a tener considerable influencia en la política estatal chilena, el objetivo era "educar al soberano", entendido como el pueblo encargado de elegir a sus representantes (Guerra, 2017; Donoso, 1961: 11-24). El decreto del ministro Manuel Montt, que creó la Escuela Normal de Preceptores en 1842, pretendía colocar la primera piedra en la conformación de un estamento docente que lograra el objetivo propuesto (Ponce, 1890: 267-270). Por sus aulas pasaría José Bernardo Suárez, alumno supernumerario de la primera generación de preceptores de la flamante escuela, regenteada precisamente por Sarmiento (Sarmiento, 1942a: 117). En este contexto, nuestro propósito principal es estudiar y poner en valor la figura del maestro normal José Bernardo Suárez en el ámbito de la educación popular chilena de la segunda mitad del siglo XIX.

Su aporte a la educación fue muy profuso durante sus noventa años de vida. Entre sus obras se cuentan numerosos artículos publicados en periódicos nacionales y extranjeros (para este último, por ejemplo Suárez, 1898a: 461-464), numerosas cartas e informes inherentes a su actividad docente y formativa, como preceptor y profesor en escuelas y liceos chilenos y luego como visitador de escuelas, redactados desde sus primeros pasos en la docencia, en la década de 1840. Labor la suya que le valió el apodo de "patriarca de la instrucción pública de Chile y América", de "primer preceptor primario", y "creador de las bibliotecas escolares", a las cuales contribuyó con su abundante producción escrita. ¿Hasta qué punto pueden suscribirse hoy estos juicios? A estos títulos se suma además la apreciación de Amanda Labarca, que lo describe como "el más notable de los visitadores de escuelas" (Labarca, 1939: 123). ¿En qué se fundamenta concretamente esta afirmación?

De su trayectoria hoy llama la atención y motiva a indagaciones la abundante literatura didáctica que escribió, lo cual le valió otro de los títulos que se le atribuyen: el de ser "el primer escritor didáctico" (Valdivia Castro, 1933: 16). Más de veinte textos de enseñanza con numerosas ediciones en el caso de varios de ellos, no solo nacionales sino también extranjeras, justificarían esta afirmación. ${ }^{3}$ Sin embargo, su obra parece haber sido escasamente

3 Rasgos notables de niños célebres de Chile contó con al menos quince ediciones detectadas, no solo en Chile, sino también en México y París (Suárez, 1898). 
analizada, por lo cual se presenta como un privilegiado campo de estudio y confrontación de modelos educativos epocales y de la actualidad (Dedieu, Enríquez, Cid, 2014: 4-8). La importancia que dicha literatura adquirió, no solo en el ámbito local sino también americano ${ }^{4}$, requiere de una revisión de sus postulados y de los modelos que de algún modo llenaba, rescatando hoy las claves de su popularidad. También suscita interés la difusión e importancia de dichas obras, registradas en las premiaciones que algunas de ellas obtuvieron en exposiciones internacionales, tanto americanas como europeas, tales como la de 1884 en Río de Janeiro y de 1889 en París (Valdivia Castro, 1933: 21). Cabe interrogarse al respecto qué impacto tuvieron los textos de Suárez en el contexto del fenómeno expansivo de la escuela pública y de una naciente cultura nacional. Recordemos al respecto que, de las más de veinte obras editadas por Suárez, al menos quince fueron adoptadas o aprobadas como textos de lectura para las escuelas públicas por el consejo universitario encargado especialmente de esta misión (por ejemplo, Suárez, 1869, 1886, 1898b).

De acuerdo a lo expuesto, puede percibirse la importancia de Suárez como educador, y de su obra, como muestra evidente de su propósito de difundir el imaginario burgués de rasgos europeos, que buscaba concretar el ideal liberal de "educar al soberano" (Sarmiento, 1900), por una parte, y por ende de confeccionar "una juventud virtuosa" (Toro Blanco, 2012).

En este contexto, nuestro estudio se centrará en uno de aquellos escritos, basado en un informe realizado por Suárez desde su labor como visitador, para la revista de educación, bibliografía y agricultura, fundada por Domingo Faustino Sarmiento y editada en Nueva York, bajo el título: Ambas Américas. A partir del mismo, se indagan ciertos aspectos de la personalidad y de la vida del maestro Suárez, en relación al paradigma de educacionista propio del ideario liberal. En segundo término, se sopesa su informe en función de su rol agente de observador y conocedor de la realidad de la educación chilena, en un momento cumbre de la historia escolar, signado ya por la vigencia de una ley y de un reglamento general que vieron la luz en 1860 y 1863 respectivamente; ambos documentos se transformaron en los pilares fundantes del ordenamiento escolar realizado por el estado docente. Por último, se analizarán sus apreciaciones acerca de diversos ámbitos del sistema de educación estatal, desde su posición de visitador de escuelas, funcionario estatal encargado de vigilar y supervisar los avances de la política educativa desde la misma institución escolar.

4 Cabe acotar que algunos, como la Guía del preceptor primario, fueron adoptados en países vecinos como Argentina (Suárez, 1869). 


\section{Suárez como "modelo" de educador y de ciudadano}

En la labor que desempeñó como educador y luego como visitador, Suárez tuvo oportunidad de interiorizarse desde dentro de los problemas que la creación de escuelas conllevaba. No en vano, según testimonio de Domingo Faustino Sarmiento, Suárez tuvo el mérito de ser "quizás el único hombre de la América del Sur que por especial vocación se haya consagrado a la Educación que se da en las escuelas..." (Sarmiento, 1867: 11). Precisamente en la presentación que Sarmiento realiza de aquel informe, el maestro comentaba las circunstancias de su encuentro con el chileno, en el marco de su función como director de la primera Escuela Normal de Santiago:

"Un día se presentó un joven barbado solicitando admisión en la escuela. Era casado i ejercía ya la profesión de maestro de Escuela en una provincia, pero quería serlo en realidad, preparándose mejor para su vocación, i dijo llamarse José Bernardo Suárez. Admitido inmediatamente, su contracción fue asidua, su conducta ejemplar, i terminó el curso con recomendación especial, habiendo además aprendido francés, para ensanchar la esfera de su conocimiento" (Sarmiento, 1867: 11).

A comienzos del mismo año este joven había solicitado al tribunal de apelaciones ser declarado pobre, posiblemente para conseguir una beca y poder continuar sus estudios (Gaceta de los Tribunales, 26-II-1842). El dato lo ubica en aquel sector social que respondió al llamado del gobierno, de baja condición económica, pero con aspiraciones a mejorar dicha condición aprovechando su formación. Entre los datos que aporta Figueroa acerca de sus estudios, destaca su asistencia a la escuela particular del maestro Juan Barrera (1830), estudios de latín en el Convento de San Francisco bajo el sistema lancasteriano, y de filosofía escolástica en el de la Merced (Iturriaga, 1989; Baeza, 2017a, 2017b, 2018; Figueroa, 1896: 27). ${ }^{5}$

En consonancia con dichas aspiraciones, en 1840, con solo dieciocho años, fundaba su propia escuela en calle San Francisco para enseñar escritura moderna, a la par que un profesor le enseñaba caligrafía inglesa, para abandonar la española, considerada en desuso (Figueroa, 1896: 28). Al año siguiente de haber ingresado a la Normal, en 1843, el joven Suárez fue destinado inmediatamente por el gobierno a la escuela municipal anexa al liceo de San Felipe (Larenas, 1865: 62), según sus mismas palabras: "en atención a la absoluta necesidad que había de personas competentes". Este hecho denota la gran estima que Sarmiento tenía de sus condiciones y la amistad

5 Otra versión habla de sus estudios de filosofía en el convento de Santo Domingo, y no de la Merced (Pérez Yañez, 1943: 357). 
que se fue forjando entre ambos, tal como queda testificado en la biografía que Suárez le dedicó en su obra Rasgos notables de hombres célebres de Chile. En ella cuenta una anécdota en particular, ocurrida en ocasión de su visita de bienvenida al argentino, cuando éste regresaba de su viaje a Europa en 1847. En esa oportunidad Suárez fue presentado por el maestro, "con toda la etiqueta de estilo i como si fuera un personaje de alta importancia al señor ministro del interior entonces don Manuel Montt", al igual que lo había hecho otras veces con argentinos de prestigio: el General Las Heras, el doctor Ocampo, entre otros; actitud explicada por el mismo autor en estos términos: "Sarmiento hacía esto con sus discípulos, no porque estuviese persuadido que valíamos algo, sino por dar importancia i realce al preceptorado, entonces abatido, humillado, envilecido" (Suárez, 1863: 283-284).

Varios de sus alumnos en aquella escuela de San Felipe serían después hombres notables de la vida pública; entre ellos se contaban Abdón Cifuentes, José Antonio Varas, Eulojio Altamirano y Nicanor Zenteno, todos vinculados a la educación pública (Figueroa, 1896: 29-30). Trasladado nuevamente a Santiago, en 1847 sería profesor de colegio y poco después del que dirigía don Rafael Minvielle. En carta enviada al ministro de Justicia e Instrucción Pública don Adolfo Ibáñez, en 1896, solicitando una pensión digna, haría referencia a ese momento de su carrera, que le había permitido conocer realidades del espectro social diferentes a la suya: "He enseñado en los colejios a los hijos de la aristocracia, y en las escuelas a los hijos del pueblo" (Figueroa, 1896: 41).

Luego de haber ejercido como visitador de escuelas en las provincias de Concepción y después de Valparaíso, en 1852 fue nombrado visitador general de escuelas, con un sueldo de 400 pesos anuales. En la mencionada carta asegura que se mantuvo "firme y perseverante en su puesto, a pesar que los anteriores sucumbieron, porque no tenía obligaciones ni vicios de ningún jénero" y "lo poco que recibía me alcanzaba para vivir pobremente, pero sin deber a nadie" (Figueroa, 1896: 42). Estas escasas referencias a su vida privada nos hacen suponer que, a pesar de ser casado, no tuvo hijos con su primera esposa, la cual probablemente habría fallecido.

En 1861 sería nombrado nuevamente visitador, esta vez de la provincia de Santiago, a pesar de que la labor le significó con los años el quebranto de su salud, según consta en los informes presentados por el inspector general al ministro del ramo (Larenas, 1865: 59). En dicho cargo se encontraba cuando escribió el informe solicitado por su antiguo maestro residente por entonces en Nueva York, y en el mismo puesto permaneció hasta su jubilación. Respecto de esta, el mismo Suárez alegaba por su sueldo que correspondía a la "mísera suma de 68 pesos", a pesar de lo cual no había dejado de trabajar en aquello que consideraba su "manía", la educación del pueblo (Figueroa, 1896: 41). 
Esa "manía" sería el aliciente para contribuir a la expansión de esa educación popular, a través de varias fundaciones de escuelas, según afirma José Domingo Cortés, en su Diccionario biográfico americano, exaltando sus méritos como:

"fundador de las escuelas del regimiento de granaderos a caballo de la brigada de policía de Santiago; de la primera escuela nocturna que, en 1848, se estableció en Valparaíso; de la primera escuela superior que, en 1857, se abrió en Santiago" (Cortés, 1876: 479).

Fundaciones a las cuales se sumaba la que había efectuado previamente del Colegio Mercantil de Santiago, en 1851 (Figueroa, 1896: 30).

Otros aspectos notables de su carrera, que lo posicionaron para ocupar un alto cargo como el de visitador general, dicen relación con el puesto de subdirector de los primeros ejercicios de maestros de la Escuela Normal, realizados en 1855, y que fueron establecidos y dirigidos personalmente por Sarmiento con la ayuda de su discípulo. Según el decreto firmado el año anterior por el ministro del ramo, don Silvestre Ochagavía, Suárez debía dirigir la marcha de los ejercicios mientras que Sarmiento daría las disposiciones conducentes a su organización. Durante todo el mes de enero los preceptores de las escuelas fiscales de la provincia de Santiago debían concurrir a la Escuela Normal para ejercitarse en los métodos de enseñanza de las distintas disciplinas que impartían, costeados por el Estado. También podían participar los de escuelas municipales y particulares a voluntad, al igual que los de otras provincias, aunque ya no con el subsidio estatal, sino por cuenta de sus respectivos empleadores (Ponce, 1890: 446-449).

A pesar de haberse jubilado en 1869, Suárez continuaría impulsando la educación pública desde la tribuna periodística. Su experiencia en este rubro le venía desde los tiempos en que redactaba el Monitor de las Escuelas Primarias, junto a su maestro Sarmiento, periódico de educación fundado por el argentino en 1852, función que siguió cumpliendo junto a Santiago Lindsay. Según afirma Cortés, durante al menos cuatro años colaboró con ellos "sin recibir remuneración alguna" (Cortés, 1876: 480).

Su afán de difundir las ideas del progreso a través de la educación se vio renovado en 1886, cuando el joven José Abelardo Núñez fundó la Revista de Instrucción Primaria, de la cual Suárez también fue redactor a partir de 1887 (Núñez, 1886: 7-11). ${ }^{6}$ Del mismo modo, participó activamente en los

6 La revista venía a cubrir el vacío dejado por la supresión del Monitor de las Escuelas Primarias, ocurrida más de veinte años antes (1865), y tuvo por objeto difundir las reformas iniciadas durante el gobierno de Domingo Santa María al sistema educativo chileno. 
actos preparatorios del Congreso General de Enseñanza Pública de 1902, desempeñándose como vocal en la Comisión de Enseñanza Primaria, dirigida por Claudio Matte, presidente a su vez de la Sociedad de Instrucción Primaria y miembro de la Facultad de Humanidades (Congreso Jeneral de Enseñanza Pública, 1904: 10-11).

Más tarde, con el mismo Núñez, formaría en 1909 el Consejo de Instrucción Primaria, que contaba entre sus integrantes a las directoras de la Escuela Normal femenina, Brígida Walker e Inés E. Brown, demostrando una vez más su presencia activa en la tarea difusora de la educación popular (Huneeus, 1909: 304-305).

Sus obras siguieron utilizándose al menos hasta 1908, según lo atestigua la Memoria ministerial de ese año, en la cual su Guía del preceptor primario continuaba en los listados de libros distribuidos a escuelas privadas como texto guía para la labor docente, junto a otros textos para formación y perfeccionamiento de los profesores, destinados probablemente a mejorar sus métodos de enseñanza (Chile, 1908: 7-8).?

Esta faceta de su actividad pedagógica, referida a la producción de textos escolares, testigos clave de la historia, los ideales e ideologías de una época, entre otros aspectos, estuvo en relación directa con la escasez de un tipo de literatura apropiado para fomentar la lectura y la cultura de la población en edad escolar. Esta problemática fue denunciada por los distintos actores implicados en ella, así como ya lo habían hecho antes intelectuales de la talla de Andrés Bello, quien en 1836 ya aconsejaba una cuidadosa selección y la distribución gratuita de los textos escolares a los niños pobres (El Araucano, 5-VIII-1836; 12-VIII-1836). Suárez mismo, como preceptor primero y luego como visitador, descubrió tempranamente esta carencia y se dedicó activamente a llenar ese vacío, tal como ya lo habían hecho su maestro Sarmiento y otros educadores notables. Su esfuerzo principal se concentró en la recopilación, traducción y síntesis, así como en la transmisión a un lenguaje sencillo y adecuado, de aquellas obras que él juzgaba convenientes para la formación de los estudiantes (Medina, 1926: 60). Cabe preguntarse, en este sentido, en dónde reside el mérito de su labor literaria, si en su criterio didáctico fundado en la difusión de modelos antropológicos clásicos rescatados de la historia universal, americana o chilena; o bien, en su labor periodística, a través de la cual buscaría plasmar sus propias experiencias didácticas, para transmitirlas a las futuras generaciones docentes (Jaksić, 1991-1992; Rivera, 2017). En este sentido, el estudio de sus contribuciones periodísticas supone también analizar las características del perfil de educador que Suárez repre-

7 La apreciación efectuada se fundamenta en varios de los títulos de los demás libros entregados (Barrau, 1869; Sarmiento, 1846; Lobos, 1904; Tapia, 1906; entre otros). 
senta, partícipe activo de la vida social y política republicana. Dicho análisis puede ser significativo, no solo para aquilatar la importancia de Suárez como profesor, sino también para considerar la lectura que el público haría de sus escritos. Justamente, el mérito de Suárez en cuanto autor fue su versatilidad para transitar desde la pedagogía al campo literario y periodístico. Esto, por lo demás, no era extraño en el siglo XIX, cuando dicha versatilidad era bastante frecuente.

Su fama de pedagogo ilustre, ya largamente conocido a estas alturas, llegaba a los lugares más extremos del país, como lo demuestra, por ejemplo, la Revista del Preceptorado de Atacama, que cita sus artículos y obras en distintas oportunidades, por ser autoridad en el ámbito educativo, "reputado escritor didáctico i antiguo pedagogo" (Sociedad Unión de Preceptores, 1895: 295). ${ }^{8}$ En el mismo sentido, las palabras de un alumno de la Escuela Normal, con ocasión de un discurso fúnebre de 1906 en honor al profesor Eduardo Rossig, son representativas del lugar que ocupaba el maestro Suárez en la memoria del preceptorado:

"Querido maestro, tu nombre pasará a la historia de la pedagogía chilena al lado de los de Sarmiento, Suárez, Abelardo Nuñez, Manuel A. Ponce i otros apóstoles de nuestra enseñanza" (Gutiérrez, 1906: 10-11).

En definitiva, la trayectoria de José Bernardo Suárez, como educador y como ciudadano, corresponde al perfil ideal de preceptor que habían forjado Sarmiento, Montt y los hermanos Amunátegui en sus escritos, tal como lo describía en 1896 Pedro Pablo Figueroa, en un tono panegirístico muy acorde con la época:

"El señor Suárez, sin apartarse de su apostolado, ha hecho la propaganda de las ideas de civilización en las instituciones, proclamando la preeminencia de la inteligencia y de las virtudes cívicas encarnadas en los hijos de la democracia" (Figueroa, 1896: 33).

Cabe acotar que, para algunos historiadores, dicho perfil tenía como trasfondo una necesidad más bien práctica, vinculada a la formación de ciudadanos votantes; de allí que el leer y escribir se constituyera en una herramienta indispensable para la estabilidad del orden republicano (Castillo, 2009).

8 En la ocasión, comentaban el último artículo publicado por Suarez (1895: 189-194). 


\section{Suárez, y su rol como observador de la realidad educacional chilena}

En el Informe dedicado a Ambas Américas, los datos concretos acerca del sistema educativo, incluyendo los establecimientos privados y especiales, además de todos los niveles, según el censo de 1865, fueron registrados y analizados por el visitador, indicando que de un total de 1.819 .028 habitantes, un quinto de ellos debería estar escolarizado; pero solo asistía alrededor del 15\% de la población en edad escolar, lo cual sumaba 54.050, de un total de 363.805 niños entre 5 y 15 años. Para Suárez, este panorama era "triste i desconsolador", ya que más de 300.000 potenciales alumnos quedaban fuera del sistema escolar. Pero el visitador sacaba otra conclusión más grave todavía; haciendo una proyección a veinte años, pensaba que dicha cifra aumentaría en 100.000 más, de los cuales saldrían los futuros delincuentes, listos para sumarse a la revuelta, y derrocar cualquier gobierno bien constituido (Suárez, 1867: 13-28). Es necesario acotar que dicha proyección, aunque acertada, se quedaba corta, pues según el censo de 1885, la población en edad escolar aumentó a 606.154 niños, en tanto que la escolarizada fue de 97.105 niños, con lo cual el crecimiento fue de 229.770 potenciales alumnos, y demostró un aumento de solo un $1 \%$ en relación a tasa de 1865 , es decir un $16 \%$ total. $^{9}$

Otra inferencia que Suárez hacía ante este problema era su posible solución, la cual se vinculaba para nuestro observador con el aumento del número de escuelas (Suárez, 1867: 16). En este sentido, cabe destacar el estudio de Macarena Ponce de León, quien ha indagado acerca del proceso de expansión del sistema de instrucción primaria estatal en la segunda mitad del siglo XIX, llegando a la conclusión que la ley de 1860, al organizar el sistema educativo sobre la base de los centro urbanos y poblados rurales de más relevancia, provocó un fortalecimiento de este sector, concentrando en él los recursos tanto económicos como humanos, lo cual desequilibró la balanza en desmedro de la población rural del país que conformaba, todavía en 1880 , un $70 \%$ del padrón demográfico. Esto coincide en parte con la mencionada proyección de Suárez, dado que aún era notoriamente bajo el porcentaje de escolarización, pues ahora dejaba fuera no solo el mundo rural, sino también: "el mundo popular urbano que había ido creciendo y cambiando como resultado de las migraciones". No obstante, demuestra también que el maestro se equivocó al pensar que el crecimiento de las escuelas sería determinante para el aumento de la escolarización, pues la inasistencia de los alumnos continuó siendo un problema, en el fondo del 
cual estaba el fenómeno de la localización errada de las escuelas, que no se ubicaban donde estaba la mayoría de la población. Según observa finalmente Ponce de León, "la inasistencia escolar se constituyó en el eje del debate político sobre la obligatoriedad de la instrucción primaria, ley promulgada finalmente en 1920" (Ponce de León, 2010: 449-486).

\section{Las escuelas normales y la formación de educadores}

En el segundo apartado de su informe, el visitador se dedicaba a poner de manifiesto la situación de las dos escuelas de formación del profesorado primario existentes en Chile hasta el momento, una para hombres y otra para mujeres. La Escuela Normal de Preceptores, instalada en Santiago, contaba con el mérito de ser la primera de su tipo en toda Latinoamérica, y la segunda en todo el continente americano. Impulsada por Domingo F. Sarmiento, quien estaba secundado en su idea por el venezolano Andrés Bello y el chileno Eduardo de la Barra (Campobassi, 1975: I, 180), la Escuela se inspiraba en la fundación de la Escuela Normal de Lexington, en Massachussets, que, según afirmaba el propio Sarmiento, imitaba el modelo prusiano admirado por Mr. Charles Brood (Sarmiento, 1866: 39-40). Sin embargo, el proyecto no hubiera llegado a concretarse sin la actitud decidida del entonces presidente de la república Manuel Bulnes, quien firmó el decreto oficial de creación el 18 de enero de 1842, junto al ministro del ramo, don Manuel Montt (Cox y Gysling, 2009: 55-74). La educación sería gratuita y los alumnos recibirían un estipendio de cien pesos mensuales para su manutención y vestuario como estipulaba el artículo $6^{\circ}$ (Montt, 1842: 7). Según afirma Iván Núñez, recién en 1848 dicha escuela comenzó su funcionamiento regular, coexistiendo en ella los alumnos de las tres generaciones correspondientes. Cada una de éstas se sometía a tres años de formación, en un sistema de trabajo escolar intensivo y con un fuerte sello de disciplinamiento (Nuñez, 2010: 135). Este último aspecto era fundamental para lograr los objetivos del gobierno, ya que, en palabras de Sarmiento, en carta al ministro de Justicia, Culto e Instrucción Pública de noviembre de 1843, había que introducir:

"un sistema de educación primaria que ha de traer por resultado cambiar la faz de la educación, en un país donde no hay generalizadas ni métodos ni ideas, ni buenos hábitos, y al contrario dificultades sinnúmero, preocupaciones arraigadas, y una rutina irracional" (Sarmiento, 1942b: 87-94).

En cuanto a la primera Escuela de Preceptoras, se fundó recién en 1854, pasados ya más de diez años de la creación de la de varones (Peña, 2000). Su fundación fue encargada por el gobierno a la religiosa Anna du Roussier, la superiora de la congregación femenina del Sagrado Corazón, 
con la intención de formar maestras que pudieran dirigir las crecientes escuelas públicas femeninas, del emergente sector social medio (De la Taille, 2011: 47-70).

De acuerdo al mismo Iván Núñez, en lo cuantitativo, el incremento del personal docente idóneo "marchó a la zaga del crecimiento del sistema de las escuelas primarias" (Nuñez, 2010: 136), lo cual explica la preocupación del visitador Suárez en este sentido, puesto que en 1867 el informe constataba una cantidad aproximada de cien alumnos varones, en tanto que la de mujeres solo contaba con poco más de la mitad. El mismo visitador reclamaba la imperiosa necesidad de "aumentar desde luego su dotación a cien alumnas por lo menos, de manera que pueda dar todos los años treinta o más preceptoras" (Suárez, 1867: 16-17).

Este déficit de profesionales en el rubro era, no obstante, contradictorio con el gasto que había generado esta inversión. Según el autor del informe, ambos establecimientos costaban ya al Estado medio millón de pesos, y de ellas habían egresado cuatrocientos profesores. Sin embargo, dicha inversión había sido absolutamente deficitaria, por cuanto la mitad de aquellos abandonaban la profesión una vez cumplidos los siete años de su contrata (Suárez, 1867: 16). Cabe acotar en este sentido que el decreto de creación de la primera Escuela Normal antes mencionado, disponía en su artículo $7^{\circ}$ la obligación por parte de los alumnos becados de "servir en una escuela que el gobierno les designe, por el término de siete años; su renta, que se arreglará a las circunstancias de cada pueblo, no bajará de trescientos pesos anuales". Esta disposición quedaba reforzada por la del artículo $8^{\circ}$, que determinaba la devolución por parte de los padres, curadores o personas bajo cuyo poder estuvieran esos jóvenes, de lo gastado por el Estado en su educación (Montt, 1842: 5-8).

¿Cuáles fueron los motivos principales de aquel déficit? Ya en tiempos del primer curso de la Normal ocurrieron catorce expulsiones, por las siguientes causas:

"Uno por robo con llaves falsas, cinco por incapacidad notoria, uno por hábito inveterado de juego, cinco por desaplicación absoluta y aversión al estudio, uno por insubordinación, uno por palabras y escritos indecentes..." (Sarmiento, 1942a: 110)

Incluso algunos de ellos no terminaron sus estudios y comenzaron a trabajar, como fue el caso del mismo Suárez, enviado por el propio Sarmiento a cubrir una vacante en San Felipe, a solicitud del Intendente de Aconcagua (Sarmiento, 1942a: 99-122). Según lo expresa el visitador en su informe, incluso aquellos que ya habían egresado se separaban de la enseñanza por diversas causas: "para entregarse a ocupaciones más lucrativas; ... porque 
no llenan sus deberes, [o bien] porque se corrompen en los pueblos a donde se les envía" (Suárez, 1867: 16).

En todo caso, nuestro observador consideraba que una de las soluciones para aumentar el número de profesores idóneos pasaría por abrir una sección de externado en ambos establecimientos (Suárez, 1867: 16). El tema de la residencia de los alumnos había generado revuelo durante las primeras generaciones de postulantes, dado que la conducta de muchos de ellos había dejado mucho que desear. Ya Sarmiento, como primer director de la Escuela Normal de Preceptores, analizaba la situación de las primeras escuelas de este tipo en Francia y advertía:

"En Chile y en el primer ensayo las consecuencias perniciosas del externado han sido aún más sensibles. Ninguna vigilancia efectiva ha podido ejercerse sobre un gran número de jóvenes: los unos están hospedados en casas poderosas a donde no puede sin herir las exigencias sociales, penetrar una inspección externa; otros alquilan piezas; cuales residen en el seno de sus propias familias, y cuales gozan de una absoluta independencia" (Sarmiento, 1942a: 99-122).

Las autoridades consideraron en general, según la sugerencia del mismo Sarmiento, que el régimen de internado sería más apropiado para el logro del objetivo de "civilizar y moralizar" a aquellos que luego deberían transmitir esta enseñanza al resto de la población escolar (Nuñez, 2010: 135). Sin embargo, una década después, Suárez se inclinaba por el régimen anterior, aunque con algunas modificaciones que optimizaran el sistema, tales como el traslado de los edificios al centro de la ciudad, y la depuración del proceso de selección de los aspirantes (Suárez, 1867: 16).

Por último, una solución de más peso pasaría por aumentar la retribución de los preceptores, puesto que una de las graves falencias del sistema era la escasez de los sueldos, lo cual generaba el abandono de la profesión. Insistía aún en este aspecto cuando trataba específicamente "las necesidades de la instrucción primaria i medios de mejorarla"; allí detallaba el sueldo que cobraban los preceptores de escuelas elementales, advirtiendo que debería aumentarse a 30 pesos anuales en vez de los 25 , que no consideraba el aumento del costo de la vida, y era insuficiente para que estos profesores mantuvieran a sus familias y a los allegados. Cabe destacar la insistencia de Suárez en este punto, que él consideraba como un paso previo fundamental para la mejora de la enseñanza, insistencia que lo impulsaba a instar a las autoridades estatales para que incluyeran una contribución especial y exclusiva para este ramo en el presupuesto. El lamento del viejo maestro coincide con la queja generalizada de los preceptores de la época, que manifestaban en reiterados casos la insuficiencia del salario de un profesor para cubrir sus gastos mínimos. 
Las palabras de Suárez en el informe son ciertamente decidoras de esta realidad:

"sea por la falta de estímulo, sea por el escaso salario que reciben, son mui raros los que alcanzan a permanecer veinte años en el preceptorado. Si se les retribuyese mejor, es claro que no abandonarían tan pronto la carrera" (Suárez, 1867: 14).

La problemática volvería a ser recordada respecto de los honorarios de los visitadores. Antiguos maestros de escuela primaria y aun secundaria, y en su mayoría ex directores de escuela, además de normalistas (Labarca, 1939: 25; Egaña, 2000: 221), los visitadores, considerados por el autor del informe como "el ojo que ve i el oído que oye en materia de escuelas", sumaban 15 en 1867, uno por cada provincia, lo cual suponía un aumento casi del triple de los existentes en 1855 (Suárez, 1867: 25). Eran elegidos por su condición de hombres respetables, por sus "honrosos antecedentes", lo cual resaltaba la queja del propio visitador de Santiago por la escasez de los honorarios y viáticos que como funcionarios recibían. Cabe hacer notar, en este respecto, la actitud humanitaria de Suárez para con dos antiguos visitadores retirados, uno de los cuales consiguió del ministro su licencia por mal estado de salud y su jubilación de 41 pesos mensuales (Suárez, 1867: 26-27).

Treinta años después de este informe el autor apelaba por sí mismo ante una situación similar que le tocaba vivir en carne propia. En carta al ministro de instrucción pública, el ya septuagenario maestro reclamaba lo siguiente:

"Tengo la convicción que he sido malamente retribuido, que mis servicios no han guardado relación con los escasos sueldos que he ganado, de 25, 30 y 83 pesos mensuales, el máximun que he tenido. Creo pues, justo que hoy, que no puedo trabajar, que tengo familia y estoi viejo i achacoso, se me asignen 3.000 pesos que es el sueldo que hoy tienen los visitadores de Santiago, menos el 75 por 100, por toda jubilación y premio. José Bernardo Suárez. Santiago, agosto de 1896" (Figueroa, 1896: 44).

\section{Sistemas y métodos de enseñanza}

Según el ideario liberal al cual adhería una porción considerable de la élite política y liberal de la segunda mitad del siglo XIX (Donoso, 1946), el nuevo ciudadano republicano debía ser una persona civilizada, cuyos rasgos relevantes, "urbanidad, refinamiento, buen gusto, así como el bagaje intelectual y moral construido por la historia del saber occidental" (Serrano, Ponce de León y Rengifo, 2012: 77-78), serían adquiridos por medio de la educación. De allí que la dicotomía entre "civilización y barbarie" contó con una presen- 
cia activa en el imaginario burgués propio de la época (Sarmiento, 1889). El dilema afectaba directamente a los sistemas y métodos de enseñanza, cuya importancia no se le escapaba al antiguo maestro de escuela. De hecho, publicaría al año siguiente del citado informe la Guía del preceptor primario i de los visitadores de escuelas, obra que, según su autor, intentaba ser un apoyo de utilidad para quienes se dedicaban a la educación de la juventud, adecuado a las costumbres, el grado de ilustración y la idiosincrasia chilena (Suárez, 1869: 8).

Suárez resaltaba en su informe el problema de la escasez de bibliografía en Chile acerca de esta temática, ya bien avanzada en países europeos. Su lucidez debe ser destacada cuando afirma un principio básico, muy en boga en la educación contemporánea: no es lo mismo saber para sí mismo que saber enseñar a otros (Suárez, 1869: 19). Este problema, eje central de las ideas pedagógicas actuales (Enkvist, 2000: 166-167; Hernández de Lamas, 2000), que colocan las metodologías de enseñanza por sobre el contenido mismo, se combinaba en la época de Suárez con otro igualmente complicado, relativo a las características propias de los alumnos chilenos, menos adelantados que los europeos, de mayor edad y con menor desarrollo intelectual. Adelantándose a las influyentes propuestas del psicólogo Jean Piaget en su estudio acerca de las etapas evolutivas del niño, el maestro chileno intuía por propia experiencia la necesidad de considerar estos aspectos para mejorar la enseñanza, dado que los métodos particulares eran poco conocidos en el medio escolar local (Suárez, 1869: 18).

Además, el informe dejaba entrever una crítica aguda al cultivo de la memoria por encima del desarrollo de la inteligencia, lo cual hoy es considerado como expresión de la educación "tradicional" heredada en el siglo XVIII, de las enseñanzas de Comenio: "enseñar todo a todos", y que tenía como base la enseñanza de mucho contenido que debía ser memorizado, fundamentalmente del ámbito de las humanidades (Suárez, 1869: 18, 23; Abbagnano y Visalberghi: 2007:305). A la par, se puede percibir bajo aquella crítica otra velada al sistema educativo colonial español, derivado precisamente del enciclopedismo que predominaba en el ámbito europeo y que privaba a los hombres americanos del raciocinio, según el pensamiento de nuestro autor; en este sentido cabe destacar su actitud tajante al afirmar que debía abandonarse este sistema, a fin de formar hombres pensantes, capaces de hablar y razonar, en forma coherente (Baeza, 2017a, 2017b, 2018). ${ }^{10}$ Tomaba como ejemplo las cámaras legislativas chilenas, cuyos miembros en su mayoría no eran capaces de expresarse ante el público, a pesar de haberse educado

10 Con respecto a los métodos de enseñanza, cabe acotar que se experimentaron varios métodos, entre los cuales está el sistema monitorial o lancasteriano. 
en los mejores colegios de la república. Proponía como método para revertir la escasa capacidad oratoria y de raciocinio de los nuevos ciudadanos, la lectura en voz alta de trozos de amena literatura, y fomentar en los alumnos la expresión escrita de sus pensamientos, pero además su experiencia docente colocaba el énfasis en la práctica constante de la lectura y la escritura, que debía partir por ser habitual en las escuelas normales (Enkvist, 2000: 171 y ss). ${ }^{11}$ En el trasfondo de esta problemática, Suárez colocaba el defecto principal de la instrucción primaria en el país, que en su opinión pecaba por ser demasiado teórica y poco práctica (Suárez, 1869: 23).

Finalizaba su informe el visitador Suárez presentando una breve reseña del estado de la instrucción secundaria y superior, la cual contenía en ese momento 4.000 alumnos entre los dos niveles, contando con el Instituto Nacional que ofrecía dos secciones, una preparatoria y otra superior o universitaria, en la cual se dictaban cátedras de derecho, medicina, arte y milicia (Serrano, 1994). El informe detallaba las cátedras existentes y la bibliografía principal que se estudiaba en cada una de ellas, haciendo referencia además a los once liceos provinciales que ofrecían enseñanza secundaria, y a otras especiales como la de minería de Copiapó, la naval, la academia militar, la escuela de artes y oficios, y las artísticas, entre otras instituciones de enseñanza. En todas ellas el gobierno invertía la misma cantidad que en la instrucción primaria, utilizada para construir edificios propios e incluso internados, como el de Medicina.

Este hecho daba pie al educador chileno para advertir a los posibles lectores de Ambas Américas acerca de la escasa racionalización de las políticas públicas en las repúblicas americanas: "En Sur-América principiamos las cosas por donde deberíamos concluirlas, construimos primero el capitel i después la base" (Suárez, 1869: 28).

\section{Conclusiones}

Luego de analizar algunos aspectos de la figura José Bernardo Suárez en función del perfil de educador vigente en su época, y su propia visión acerca de ciertos indicadores del estado de la educación chilena en 1867, consideraremos a continuación los principales resultados del estudio efectuado.

En primer lugar, la figura de este educador posee varias facetas que pueden percibirse a partir de su formación humana y profesional, su carrera pedagógica y administrativa, su labor periodística y social, y por último su

11 La investigadora sueca Inger Enkvist considera el reforzamiento de la lectura y el lenguaje como parte del éxito del modelo educativo finlandés (El Mercurio, 13-VI-2010). 
alineamiento con el ideal político de corte liberal, propio de la época. A partir de este marco, destacamos su asistencia a un colegio particular en vez de parroquial o conventual; sus conocimientos de latín y de filosofía, que demuestran sus aspiraciones para elevar su condición social a través de sus propios méritos; preocupación que lo llevó también a declararse pobre ante el Tribunal de Apelaciones, posiblemente para postular a una beca de estudio de la futura Escuela Normal, creada en el mismo año de su solicitud. Respecto de su formación y su carrera pedagógica, cabe resaltar su asistencia al primer curso de la Normal, lo cual marcaría su carrera, tanto pedagógica como administrativa y periodística, pues desde entonces contaría con el apoyo de su maestro Domingo Faustino Sarmiento para avanzar en la misma. Desde su incorporación a la escuela anexa de San Felipe, en 1843, antes de terminar su curso en la mencionada escuela, hasta sus intervenciones en el Monitor de Escuelas y en otros periódicos de la época, sus contactos ubicados en los demás países americanos, nos impulsan a evaluar la influencia que Sarmiento tuvo en la extensa trayectoria de su discípulo.

Las apreciaciones que el pedagogo realizó sobre los aspectos más importantes del sistema estatal se pueden sintetizar en tres focos fundamentales. El primero se basó en la relación entre la expansión de las escuelas y el aumento de las matrículas, la cual no fue proporcional al crecimiento de la población. Según el censo de 1865, de los habitantes de Chile en edad escolar, solo asistía un 15\%, porcentaje que había aumentado apenas un $1 \%$ en veinte años, en tanto que la potencial población escolar se había casi duplicado. Pero la solución sugerida por el visitador acerca de aumentar el número de escuelas en la práctica no resultó, pues el crecimiento dependía de la demanda, y ésta de los municipios y zonas más pobladas que solicitaban las fundaciones. Este fenómeno, según lo observado en el estudio de Ponce de León, dejó fuera de la escolarización formal a la mayoría de la población del país, que en el momento de redacción del informe habitaba en zonas rurales y no urbanas.

El segundo foco afectaba, a su vez, la relación entre el aumento de matrícula y el de los profesores idóneos para la enseñanza. La condición de normalista que Suárez ostentaba lo había llevado a tomar posición en favor de esta formación, de tal modo que, al comparar los profesores antiguos con los egresados de la Escuela Normal, identificaba las cualidades de los primeros como completamente deficientes, pues no poseían habilidades para mantener la disciplina, ni para motivar a sus alumnos, ni menos aún lograban que comprendieran los contenidos que se les entregaban (Suárez, 1906a: 52 y 1906b: 81). Estos argumentos le servían para defender el sistema normalista, aunque sugería modificaciones que apuntaban principalmente a restaurar la antigua modalidad del externado en forma paralela al internado, adaptándolo no obstante a las circunstancias del momento. 
Por último, el tercer foco vinculaba ignorancia con delincuencia. Se trataba de un argumento de peso para la élite gobernante, que pretendía consolidar el orden republicano incorporando el mayor número posible de habitantes a la escuela, puesto que "educar al soberano" equivalía a garantizar el orden, disminuyendo la delincuencia o las revueltas populares y, en definitiva, luchar contra la "barbarie" para edificar la "civilización". Colocar el énfasis en dicho argumento demostraba una vez más la adhesión fiel de Suárez al ideario liberal predominante en la época.

Respecto de la Escuela Normal como pilar esencial del sistema de formación del profesorado, nuestro autor advertía a las autoridades gubernamentales acerca de la inversión realizada hasta el momento en la formación de preceptores, y el déficit que dicha inversión suponía para las arcas del Estado, toda vez que casi un $50 \%$ dejaba la profesión por sueldos insuficientes para el mantenimiento de una familia. Según el pensamiento de Suárez, ante esta desproporción, se imponía la necesidad de echar mano al presupuesto nacional, fijando un ítem especial relativo al aumento de remuneraciones para el profesorado, lo cual preanunciaba una súplica del gremio que tendría consecuencias trascendentales, transformándose en el talón de Aquiles de las políticas públicas en el rubro de la educación.

El tercer aspecto que seleccionamos del informe de José Bernardo Suárez se deriva del anterior, puesto que si los profesores de la Escuela Normal eran los más demandados por los municipios desde los lugares más recónditos de Chile, parece evidente que su idoneidad era superior a la de los antiguos maestros de primeras letras. En este sentido, el pedagogo chileno centraba su atención en los métodos de enseñanza que aquellos debían implementar en la práctica. Como buen discípulo de Sarmiento, renegaba del aprendizaje memorístico, ligado al enciclopedismo dieciochesco, y por propia experiencia profesional criticaba dicha metodología, señalando la importancia del fomento de la lectura, de la práctica de leer en voz alta y de la comprensión de lo que se leía, en aras a formar alumnos pensantes, con capacidad de argumentar y de expresar coherentemente sus ideas en público; aspecto este último, que nos recuerda una vez más la filiación de Suárez al pensamiento decimonónico, en cuanto retomaba los lineamientos políticos, jurídicos y culturales de la antigua república romana (Huidobro, 2015).

En este contexto, consideramos fundamental resaltar, por último, el perfil de Suárez, con sus múltiples facetas de preceptor de escuela, profesor de liceo, visitador de escuelas y, por tanto, funcionario público, como periodista, participando activamente en la prensa de carácter pedagógico y general, en fin, como escritor didáctico. Esta versatilidad lo vincula a un ideal "de hombre, de ciudadano i de educador", en palabras de Fernández Peña, impulsado desde la propia Escuela Normal de corte sarmientino. De una moralidad "intacha- 
ble" y con habilidades "invaluables" para desempeñar su rol de educador con vocación y tesón (Sarmiento, 1867: 13-14), fue reconocido por muchos de aquellos contemporáneos suyos que dejaron testimonio de su valía, por haberlo conocido personalmente: desde el mismo Sarmiento, pasando por José Domingo Cortés, Pedro Pablo Figueroa, José Abelardo Núñez, Manuel Antonio Ponce, y José María Muñoz Hermosilla, quien también le dedicó páginas elogiosas en su obra acerca de la Historia elemental de la pedagojía chilena (Muñoz Hermosilla, 1918), y luego en conferencias realizadas con ocasión de su muerte; hasta el discurso fúnebre, pronunciado por don Carlos Fernández Peña, presidente de la Asociación de Educación Nacional, de la cual Suárez fue director vitalicio. En definitiva, su figura entronca con el "modelo de ciudadano" clásico, que los intelectuales de la época pretendieron impulsar para garantizar el orden republicano de reciente creación, modelo que el educador popular chileno pretendió plasmar a través de su labor educativa, periodística y literaria, demostrando su alto concepto de lo que debía ser un maestro (Cruz, 2002: 27-37). Los discípulos que formó desde sus primeros pasos en la escuela anexa de San Felipe, hasta sus últimos días, dieron muestras del ideal de excelencia propio de aquella educación clásica que Suárez pretendió transmitir a través de sus enseñanzas y de su propia vida. Abdón Cifuentes, José Antonio Varas, Benjamín Vicuña Mackenna, y Arturo Prat, entre tantos otros, fueron algunos de los futuros "hombres notables de Chile" que recibieron sus enseñanzas. Las palabras conclusivas del discurso fúnebre antes mencionado expresan claramente que el ideal heroico de la antigua educación clásica estaba aún vigente en 1912 entre sus admiradores:

"[La Asociación de educación nacional] quiere ver a este gran maestro que ha volado también en una serena mañana del mes de Mayo, a reunirse con su amado alumno de la Escuela de la Campana, Arturo Prat, unidos ambos en un glorioso monumento en que el maestro señalándole el norte lo guíe para ser el 21 de Mayo de 1879 el mas grande de nuestros héroes i el mas austero i virtuoso de nuestros conciudadanos..." (Fernández Peña, 1912: 167).

\section{Referencias bibliográficas}

\section{Fuentes primarias}

\section{a) Fuentes documentales}

Chile (1908). Memoria del Ministerio de Instrucción Pública presentada al Congreso Nacional en 1908. Santiago: Imprenta Cervantes.

Congreso Jeneral de Enseñanza Pública (1904). Congreso Jeneral de Enseñanza Pública de 1902. Actas i trabajos; actos preparatorios; secciones jenerales; sec- 
ción de instrucción primaria. Santiago: Imprenta y Litografía i Encuadernación Barcelona.

Cortés, J. D. (1876). Diccionario biográfico americano. París: Tipografía Lahure (1ª edición, 1875).

Fernández Peña, C. (1912). "Necrolojía", en Revista de Educación Nacional, antes "La Revista Pedagójica", Vol. VIII, № 3, pp. 165-167.

Huneeus, J. (1909). "Acta de Creación del Consejo de Instrucción Primaria, 5 de junio de 1909, según Art. $1^{\circ}$ del Decreto correspondiente", en Revista de Instrucción Primaria, Año XXIII, Nº 6, pp. 304-305.

Larenas, A. (1865). Informe que el inspector general de instrucción primaria presenta al señor Ministro de Instrucción pública correspondiente al año escolar de 1864. Santiago: Imprenta Nacional.

Montt, M. (1842). "Decreto del Ministro de Justicia Culto e Instrucción Pública. Escuela Normal. Santiago, 18 de enero de 1842", en Boletín de las leyes y de las órdenes y decretos del gobierno. Vol. X. Santiago: Imprenta de la Independencia, pp. 5-8.

Ponce, M. A. (1890). Prontuario de lejislación escolar: Recopilación de leyes, decretos, circulares i resoluciones sobre instrucción primaria. Santiago de Chile: Imprenta Ercilla.

Sarmiento, D. F. (1846). Instrucción para los maestros de escuela: para enseñar a leer por el método gradual de lectura. Santiago: Imprenta de los Tribunales.

Sarmiento, D. F. (1866). Las escuelas. Base de la prosperidad i de la República en los Estados Unidos. Informe al ministro de Instrucción pública de la República Arjentina. Nueva York: Edward F. Davidson.

Sarmiento, D. F. (1867). "José Bernardo Suárez de Chile", en Ambas Américas, revista de educación, bibliografía i agricultura bajo los auspicios de Domingo F. Sarmiento, Vol. I, № 2, pp. 13-14.

Sarmiento, D. F. (1889). Civilización y barbarie: vidas de Quiroga, Aldao i el Chacho. Buenos Aires, Lajouane.

Sarmiento, D. F. (1900). Educar al soberano. Buenos Aires: Imprenta y Litografía "Mariano Moreno".

Sarmiento, D. F. (1942a). "Memoria correspondiente al año 1843. Escuela Normal. Santiago, enero 4 de 1844", en Ministerio de Educación Pública: Sarmiento director de la Escuela Normal. 1842-1845. Santiago: Imprenta Universitaria.

Sarmiento, D. F. (1942b). "Nueva fórmula de contrato con los alumnos. Escuela Normal. Noviembre de 1843", en Ministerio de Educación Pública: Sarmiento director de la Escuela Normal. 1842-1845. Santiago: Imprenta Universitaria. 
Suárez, J. B. (1863). Rasgos biográficos de hombres notables de Chile. Santiago: Imprenta Nacional.

Suárez, J. B. (1867). "Reseña sobre el estado actual de la instrucción primaria en Chile", en Ambas Américas, revista de educación, bibliografía i agricultura bajo los auspicios de Domingo F. Sarmiento, Vol. I, № 2, pp. 15-28

Suárez, J. B. (1869). Guía del preceptor primario i del visitador de escuelas: Obra protejida por el Gobierno de la República Argentina para texto de enseñanza en aquel país. Santiago: Imprenta de la Unión Americana ( $1^{\text {a }}$ ed. 1868).

Suárez, J. B. (1886). Guía del preceptor primario i del visitador de escuelas: Obra protejida por el Gobierno de la República Argentina para texto de enseñanza en aquel país, aprobada en Chile con el mismo fin por el Consejo Universitario, i recomendada al Supremo Gobierno para que proteja su impresión. Santiago: Imprenta de la Unión Americana ( $1^{\text {a }}$ ed. 1868).

Suárez, J. B. (1895). "Mejoras hechas en la Instrucción primaria desde la fundación de la primera Escuela Normal del Preceptores hasta el presente", en Revista de Instrucción Primaria, pp. 189-194.

Suárez, J. B. (1898a). "Educación popular", en Revista de la Instrucción Pública de Colombia, № 7 (47), pp. 461-464.

Suárez, J. B. (1898b). Rasgos notables de niños célebres. París: Librería de la Vda. de Ch. Bouret. (1 ${ }^{\text {a }}$ ed, i1859?).

Suárez, J. B. (1906a). "Lo que eran las escuelas antes de la fundación de la Normal en 1842", en La Educación Nacional. Mensuario pedagójico. Órgano de la Escuela Normal de preceptores de Santiago, № III, pp. 51-59.

Suárez, J. B. (1906b). "Lo que eran las escuelas antes de la fundación de la Normal en 1842", en La Educación Nacional. Mensuario pedagójico. Órgano de la Escuela Normal de preceptores de Santiago, № III, pp. 80-82.

\section{b) Publicaciones periódicas}

Ambas Américas: Revista de educación, bibliografía i agricultura, bajo los auspicios de Domingo F. Sarmiento. Nueva York, 1867-1868.

El Araucano, Santiago, 1836.

Gaceta de los Tribunales, Santiago, 1842.

La Revista del Preceptorado de Atacama, Copiapó, 1893-1900.

Monitor de las Escuelas Primarias, Santiago, 1852.

Revista de Instrucción Primaria, Santiago, 1866-1867.

El Mercurio, Santiago, 13-VI-2010. 


\section{Fuentes secundarias}

\section{a) Artículos y capítulos de libros}

Baeza, A. (2017a). "Educational Reform, Political Change, and Penury: Primary Schooling and the Monitorial System of Education in Chile, approx. 1810-1833", en M. Caruso (editor), Classroom Struggle. Organizing Elementary School Teaching in the 19th Century. Frankfurt am Main: Peter Lang.

Baeza, Andrés. (2017b). "One Local Dimension of a Global Project: The Introduction of the Monitorial System of Education in Post-Independent Chile, 1821-1833", en Bulletin of Latin American Research, Vol. 36, № 3, pp. 340-353.

Baeza, A. (2018). "Bosquejo compendioso del sistema de enseñanza mutua", en Cuadernos chilenos de historia de la educación, №9, pp. 180-186.

De la Taille, A. (2011). "Ana du Roussier: portadora de la pedagogía del sagrado corazón por el mundo. Pensamiento Educativo", en Revista de Investigación Educacional Latinoamericana, Vol. 34, № 1, pp. 355-376.

Dedieu, J. P., L. R. Enríquez, y G. Cid. (2014). "Fabricación heroica y construcción de la memoria histórica chilena (1844-1875)", en Cahiers du monde hispanique et luso-brésilien, № 104, pp. 47-70.

Donoso, R. (1961). "La labor educativa y literaria de Sarmiento en Chile", en Unión de Universidades de América Latina (Buenos Aires), $2^{\text {da }}$ ss., Vol. 1, N 4, pp. 11-24.

Egaña, M. L. y M. Monsalve. (2006). "Civilizar y moralizar en la escuela primaria popular", en Rafael Sagredo y Cristián Gazmuri (dir.), Historia de la vida privada en Chile. Tomo II: El Chile moderno de 1849 a 1925. Santiago: Taurus-Aguilar chilena ediciones.

Gutiérrez A. R. (1906). "Discurso del alumno de quinto año de la Escuela Normal de Preceptores", en La Educación Nacional. Mensuario pedagójico. Órgano de la Escuela Normal de preceptores de Santiago, № III, pp. 10-11.

Huidobro, G. (2015). "La recepción de los clásicos durante las independencias hispanoamericanas: propuesta para una aproximación teórica e historiográfica", en Intus-Legere Historia, Vol. IX/1, pp. 47-68.

Jaksić, I. (1991-1992). "Sarmiento y la prensa chilena del siglo XIX", en Historia $N^{\circ} 26$, pp. 117-144.

Núñez, I. (2010). "Escuelas normales: una historia larga y sorprendente. Chile (1842-1973)", en Revista Pensamiento Educativo, № 46-47, pp. 133-150.

Núñez, J. A. (1886). "La Revista de Instrucción Primaria", en Revista de Instrucción Primaria, $N^{\circ} 1 / 1$, pp. 7-11. 
Ossa, J. L. (2017). "Revolución y construcción republicana en Chile, 1810-1851", en I. Jaksić y J. L. Ossa (editores), Historia política de Chile, 1810-2010. Tomo I: Prácticas políticas. Santiago: Fondo de Cultura Económica.

Ponce de León, M. (2010). "La llegada de la escuela y la llegada a la escuela. La extensión de la educación primaria en Chile, 1840-1907", en Historia N43, Vol. 2, pp. 449-486.

Rivera C. (2017). "Prensa y política. El poder de la construcción de la realidad. Chile, siglos XIX y XX", en I. Jaksić y J.L. Ossa (editores), Historia política de Chile, 1810-2010. Tomo I: Prácticas políticas. Santiago: Fondo de Cultura Económica.

Sociedad Unión de Preceptores. (1895). "Juicio honroso", en La Revista del Preceptorado de Atacama, Vol. III, № 2, p. 295.

Toro, P. (2012). "Dimensiones de la confección de una juventud virtuosa: manuales de urbanidad en Chile (c.1840-c.1900)", en Universum, №27 (1), pp. 191-205.

\section{b) Libros}

Abbagnano, N. y A. Visalberghi. (2007). Historia de la pedagogía. México: Fondo de Cultura Económica (1ª edición, 1954).

Barrau, T. (1869). Dirección moral para los institutores. Santiago: Imprenta Nacional.

Campobassi, J. S. (1975). Sarmiento y su época. 2 vols. Buenos Aires: Editorial Losada.

Castillo, V. (2009). La creación de la República: la filosofía pública en Chile, 18101830. Santiago, LOM.

Cox, C. y Gysling, J. (2009). La formación del profesorado en Chile 1842-1987. Santiago: Universidad Diego Portales.

Cruz, N. (2002). El surgimiento de la educación secundaria pública en Chile: 1843-1876 (el plan de estudios humanista). Santiago: Centro de Investigaciones Diego Barros Arana.

Donoso, R. (1946). Las ideas políticas en Chile. México: Fondo de Cultura Económica.

Egaña, L. (2000). La educación primaria popular en Chile. Santiago: Dirección de Biblioteas, Archivos y Museos y Centro de Investigaciones Diego Barros Arrana.

Enkvist, I. (2000). La educación en peligro. Madrid: Grupo Unisón Ediciones.

Figueroa, P. P. (1896). La historia de un maestro: el silabario y la escuela. Santiago: Imprenta Porteña. 
Guerra, J. (2017). Sarmiento: su vida y sus obras. Prólogo de Jorge Pinto Rodríguez e introducción Marcelo Aristeo. Santiago: Volantines Ediciones (1ª edición, 1901).

Hernández de Lamas, G. (2000). Los desafíos del aprendizaje. Buenos Aires: Editorial Baraga.

Iturrriaga C. R. (1989). Las escuelas franciscanas. Santiago: Publicaciones del Archivo Franciscano.

Labarca, A. (1939). Historia de la enseñanza. Santiago: Imprenta Universitaria.

Lobos A. F. (1904). Juicios críticos sobre los principales métodos caligráficos. Santiago: Imprenta, Litografía i Encuadernación Esmeralda.

Medina, J.T. (1926). Biblioteca chilena de traductores: 1820-1924. Santiago: Establecimientos Gráficos de Balcells.

Muñoz H., J.M. (1918). Historia elemental de la pedagojía chilena. Santiago: Casa Editorial Minerva.

Pérez Y., R. (1943). Forjadores de Chile. 2 vols. Santiago: Antera.

Serrano, S. (1994). Universidad y nación: Chile en el siglo XIX. Santiago: Universitaria.

Serrano, S.; M. Ponce de León y F. Rengifo. (2012). Historia de la Educación en Chile (1810-2010): Tomo I. Aprender a leer y escribir (1810 - 1880). Santiago: Taurus.

Tapia R., R. (1906). Guía o nuevo Método para la enseñanza de la escritura Derecha compuesto para las Escuelas i colejios de la República. Santiago: Impr., Lito. i Encuadernación Barcelona (1ª ed. 1900).

Valdivia C., C. (1933). Rápida mirada al panorama de la obra del primer preceptor primario y escritor didáctico don José Bernardo Suarez. Santiago: Imprenta Renovación.

\section{c) Tesis}

Peña T., M. (2000) Hijas amadas de la patria. Historia de la Escuela Normal de Preceptoras de Santiago, 1854-1883. Tesis para optar al grado académico de Licenciado en Historia. Santiago: Pontificia Universidad Católica de Chile.

\section{Cómo citar este artículo:}

Soaje de Elías, R. y M. Salas Fernández (2018) "José Bernardo Suárez: un agente de la educación popular y su visión sobre la instrucción primaria en Chile (1867)", en Revista de Historia y Geografía, №39, pp. 49-72, Escuela de Educación en Historia y Geografía, Universidad Católica Silva Henríquez (UCSH), Santiago de Chile.

[http://ediciones.ucsh.cl/ojs/index.php/RHyG/article/view/1692/1554] 